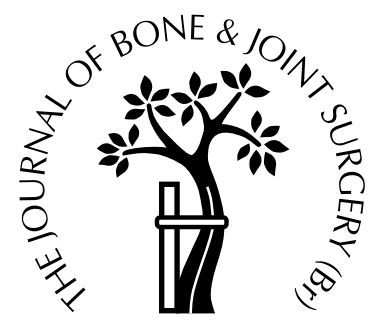

\title{
Displaced fractures of the hip in children
}

\author{
MANAGEMENT BY EARLY OPERATION AND IMMOBILISATION \\ IN A HIP SPICA CAST
}

\author{
J. M. Flynn, K. L. Wong, G. L. Yeh, J. S. Meyer, R. S. Davidson \\ From the Children's Hospital of Philadelphia, USA
}

$\mathbf{F}$ ractures of the hip in children have been associated with a very high rate of serious complications including avascular necrosis (up to 47\%) and coxa vara (up to $32 \%$ ). Over a period of 20 years, we have treated displaced fractures by early anatomical reduction, internal fixation and immobilisation in a spica cast to try to reduce these complications.

We have reviewed 18 patients who had a displaced non-pathological fracture of the hip when under 16 years of age. Their mean age at the time of the injury was eight years ( 2 to 13 ). They returned for examination and radiography at a mean follow-up of eight years ( 2 to 17). Each patient had been treated by early (" 24 hours) closed or open reduction with internal fixation and $\mathbf{1 6}$ had immobilisation in a spica cast.

By Delbet's classification, there was one type-I, eight type-II, eight type-III, and one type-IV fractures. There were no complications in 15 patients. Avascular necrosis occurred in one patient (type-III), nonunion in one (type-II, one of the two patients who did not have a cast) and premature physeal closure in one (type-I). There were no cases of infection or complications as a result of the cast.

Our treatment of displaced hip fractures in children by early reduction, internal fixation, and immobilisation in a spica cast gave reduced rates of complications compared with that of large published series in the literature.

J Bone Joint Surg [Br] 2002;84-B:108-12.

Received 8 December 2000; Accepted after revision 23 May 2001

J. M. Flynn, MD, Staff Orthopaedic Surgeon

R. S. Davidson, MD, Staff Orthopaedic Surgeon

Division of Orthopaedic Surgery

J. S. Meyer, MD, Radiologist

Department of Radiology

The Children's Hospital of Philadelphia, 2 Wood Centre, 34th Street and

Civic Centre Boulevard, Philadelphia, Pennsylvania 19104, USA

K. L. Wong, MD, Postgraduate Year 4 Resident

G. L. Yeh, MD, Postgraduate Year 4 Resident

Department of Orthopaedic Surgery, Hospitals of the University of Pennsylvania, Silverstein Building, Second Floor, 3400 Spruce Street, Philadelphia, Pennsylvania 19104, USA.

Correspondence should be sent to Dr J. M. Flynn.

(C)2002 British Editorial Society of Bone and Joint Surgery 0301-620X/02/111972\$2.00
Unlike adults, healthy children rarely sustain a fracture of the femoral neck the incidence of which is less than $1 \%$ of all paediatric fractures. It is usually the result of highenergy injury. Previous studies have reported a high rate of complications, including avascular necrosis, coxa vara, premature physeal closure, nonunion and infection. ${ }^{1-6}$

Colonna ${ }^{7}$ adapted a classification system described by Delbet, which identifies four types of hip fracture in children, based on the location of the line of the fracture. This classification is widely accepted and is used to determine the treatment and prognosis (Fig. 1). Type-I fractures are transepiphyseal separations, with or without dislocation of the femoral head from the acetabulum, and type II are transcervical, type III basicervical and type IV intertrochanteric fractures.

Although most published series have a high rate of complications several authors ${ }^{8-10}$ have described improved results with aggressive treatment consisting of urgent reduction and internal fixation. For displaced fractures in children, our standard protocol has been anatomical closed reduction, internal fixation and immobilisation in a cast. We now report the results of our experience over 20 years.

\section{Patients and Methods}

We reviewed retrospectively every displaced, non-pathological fracture of the hip in children under the age of 16 years which had presented to our centre in the past 20 years. There were 22 patients, of whom we were able to contact 18 . Of the four who were lost to follow-up, two had no evidence of complications 24 months after injury, and in two the follow-up was so short that no conclusions could be drawn. We reviewed the records and radiographs of the remaining 18. Follow-up clinical examination, radiological evaluation and assessment of function using the Iowa hip score $^{11}$ were carried out at least two years after the fracture. We also reviewed other available studies such as MRI, bone scans and scanograms.

All 18 patients had urgent closed reduction and internal fixation within 24 hours of the injury. Sixteen had additional immobilisation in a cast for six weeks. Open reduction was performed using an anterior approach if anatomical reduction was not possible by closed means under fluoroscopic imaging. Hip screws were used for most 

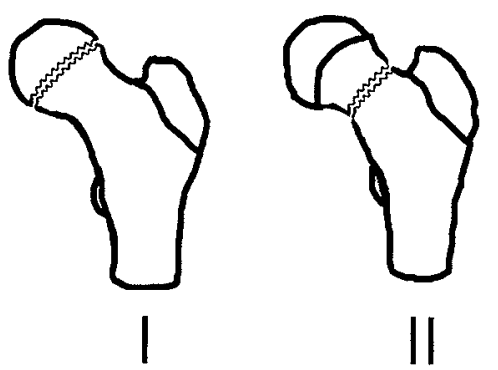

II

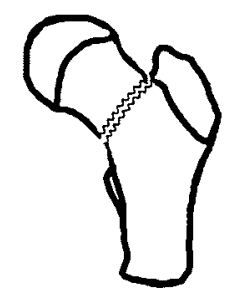

III

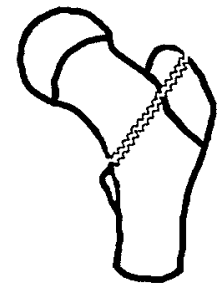

IV
Fig. 1

Diagram showing the classification of fractures of the hip in children as modified by Colonna from the classification of Delbet: type I, transepiphyseal; type II, transcervical; type III, basicervical; and type IV, intertrochanteric (pertrochanteric). of the older (13) and Steinmann pins for the younger patients (4) (Table I and Fig. 2). In one older patient with an intertrochanteric fracture, dynamic hip screws and a sideplate were used. Except in type-I transepiphyseal fractures, no fixation devices crossed the physis. After operation, all patients remained non-weight-bearing for a minimum of six weeks, followed by progressive weightbearing in uncomplicated cases. When Steinmann pins were used, they were removed at a mean of two months after injury, when radiological union had been confirmed. In patients with a considerable amount of growth remaining, the implants were removed at a mean of 22 months after the operation.

\section{Results}

There were ten boys and eight girls with a mean age of eight years (2 to 13). The left hip was involved in ten and the right in eight. The mean follow-up was for seven years (2 to 17). Using Delbet's classification, there was one typeI (6\%), eight type-II (44\%), eight type-III (44\%), and one type-IV fractures (6\%). Most were the result of high-energy injury, and all were significantly displaced. Four fractures had been caused by a motor-vehicle accident, five following impact with a vehicle, and eight by a fall. One patient sustained a type-II fracture when a branch of a tree fell on her.

Table I. Details of the 18 patients with displaced fracture of the hip

\begin{tabular}{|c|c|c|c|c|c|c|c|c|}
\hline Case & $\begin{array}{l}\text { Age } \\
(\mathbf{y r})\end{array}$ & Gender & Mechanism & Treatment* & Hip score & $\begin{array}{l}\text { Follow-up } \\
(\text { yrs })\end{array}$ & $\begin{array}{l}\text { Type of } \\
\text { fracture } \\
\text { (Delbet) }\end{array}$ & Complications \\
\hline 1 & 11 & M & Fall & $\begin{array}{l}\text { CR, Steinmann } \\
\text { pin fixation }\end{array}$ & 100 & 7.5 & III & None \\
\hline 2 & 4 & M & Car $v$ pedestrian & OR, screw fixation & 100 & 17 & II & None \\
\hline 3 & 13 & M & $\mathrm{MVA} \dagger$ & CR, screw fixation & 96 & 3 & I & Leg shortening $2.5 \mathrm{~cm}$ \\
\hline 4 & 5 & $\mathrm{~F}$ & Car $v$ pedestrian & $\begin{array}{l}\text { CR, Steinmann } \\
\text { pin fixation }\end{array}$ & 100 & 5 & II & None \\
\hline 5 & 12 & M & Fall & CR, screw fixation & 100 & 2.5 & III & None \\
\hline 6 & 11 & M & Fall & CR, screw fixation & 90 & 5 & III & Type-2 AVN \\
\hline 7 & 4 & $\mathrm{~F}$ & Car $v$ pedestrian & $\begin{array}{l}\text { CR, Steinmann } \\
\text { pin fixation }\end{array}$ & 100 & 13.5 & II & None \\
\hline 8 & 8 & M & Fall & $\mathrm{CR}$, screw fixation & 100 & 9 & II & None \\
\hline 9 & 9 & $\mathrm{~F}$ & Car $v$ pedestrian & $\begin{array}{l}\text { CR, screw and } \\
\text { sideplate }\end{array}$ & 100 & 2 & IV & None \\
\hline 10 & 9 & M & MVA & CR, screw fixation & 100 & 10.5 & III & None \\
\hline 11 & 8 & M & Tree branch fell & $\mathrm{CR}$, screw fixation & 100 & 6 & II & None \\
\hline 12 & 2 & $\mathrm{~F}$ & Fall & $\begin{array}{l}\text { CR, Steinmann } \\
\text { pin fixation }\end{array}$ & 100 & 12 & II & None \\
\hline 13 & 9 & $\mathrm{~F}$ & MVA & OR, screw fixation & 96 & 8 & III & None \\
\hline 14 & 9 & M & Fall & $\mathrm{CR}$, screw fixation & 100 & 12 & II & None \\
\hline 15 & 5 & $\mathrm{~F}$ & Fall & OR, screw fixation & 100 & 4 & III & None \\
\hline 16 & 5 & $\mathrm{~F}$ & Fall & CR, screw fixation & 100 & 3 & III & None \\
\hline 17 & 9 & M & MVA & OR, screw fixation & 100 & 13 & III & None \\
\hline 18 & 11 & $\mathrm{~F}$ & Truck $v$ pedestrian & OR, screw fixation & 71 & 4 & 11 & $\begin{array}{l}\text { Nonunion } \\
\text { Leg shortening of } 3.0 \mathrm{~cm}\end{array}$ \\
\hline
\end{tabular}

* CR, closed reduction; OR, open reduction

$\dagger$ motor-vehicle accident 


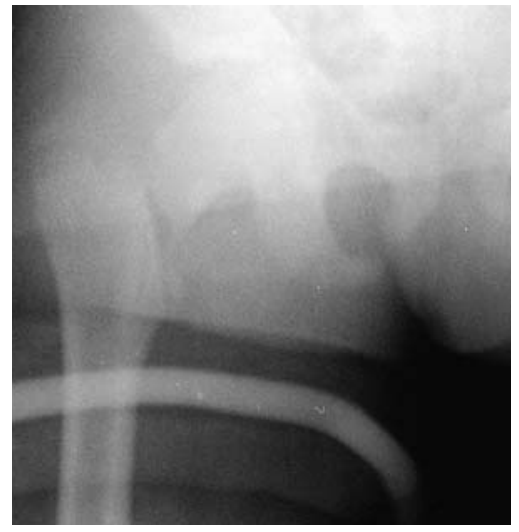

Fig. 2a

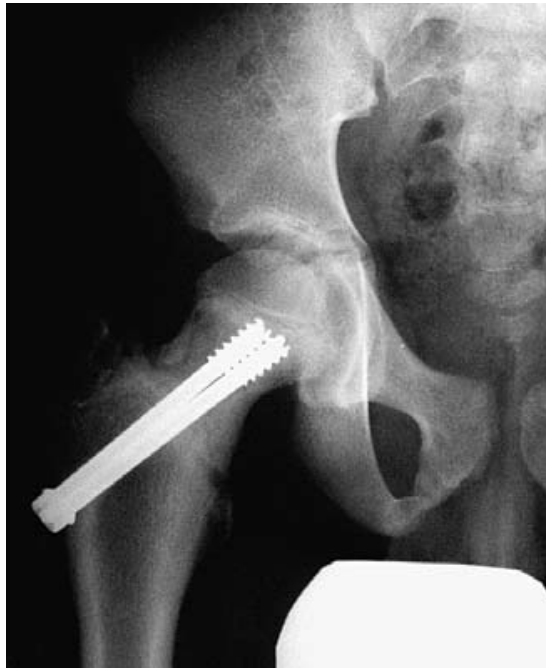

Fig. 2c

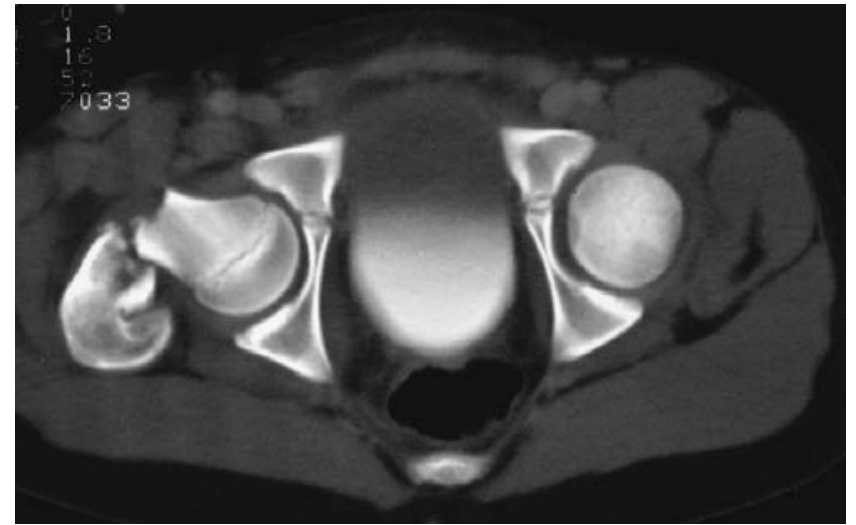

Fig. 2b

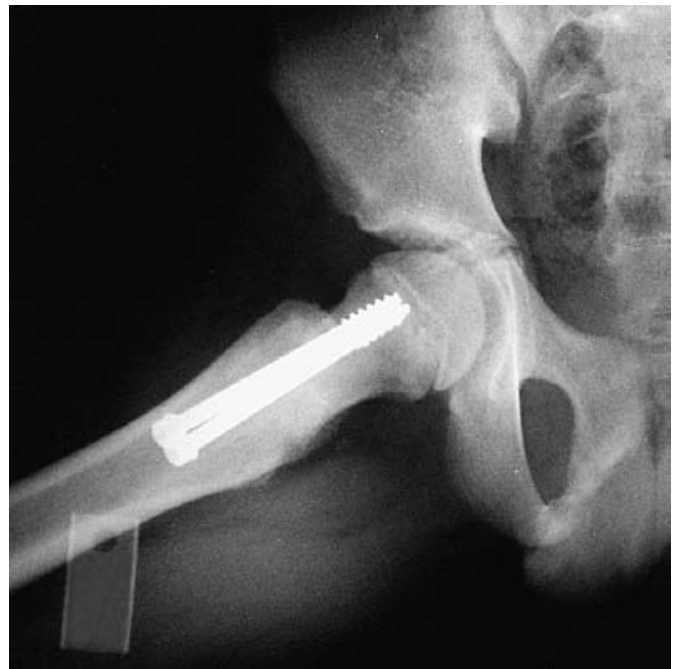

Fig. 2d

Case 1. Figure 2a - Preoperative radiograph of an 11-year-old boy who sustained a hip fracture from a fall showing a Delbet type-III basicervical fracture. Figure $2 b-$ CT of the right hip showing displacement of the fracture. Figures $2 \mathrm{c}$ and $2 \mathrm{~d}-$ Postoperative anteroposterior (c) and lateral (d) radiographs at seven months showing healing of the fracture.

Fifteen patients $(83 \%)$ had no complications from the fracture and returned to full function with a normal range of movement of the hip. There were no cases of infection or complications relating to the cast. The mean Iowa hip score was 97 out of 100 (71 to 100). One patient (case 6) developed avascular necrosis of the femoral head (Fig. 3). He had a type-III basicervical fracture which was treated by two cannulated screws and a hip spica. Twenty-two months after the operation, he required an adductor release and Petrie casting for a persistent adduction contracture. Five years after surgery, the avascular necrosis had not resulted in significant collapse, and he complained only of mild hip pain during rigorous sporting activity.

One patient (case 18), who had been hit by a truck, sustained an ipsilateral fracture of the pelvis and a type-II fracture of the femoral neck. The latter was fixed by screws and no cast was used. Nonunion developed, which required a graft from the iliac crest, further fixation by a hip screw and advancement of the greater trochanter for weakness of the abductor muscles. The fracture eventually healed after 26 months but with a leg-length disrepancy of $3 \mathrm{~cm}$. At the latest follow-up at 49 months, the hip had $140^{\circ}$ flexion, $30^{\circ}$ abduction $\left(20^{\circ}\right.$ less than the unaffected side), and $15^{\circ}$ internal rotation $\left(30^{\circ}\right.$ less than the unaffected side). The patient was, however, able to undertake most activities with only occasional pain.

Three patients had a leg-length discrepancy greater than $1.5 \mathrm{~cm}$. One (case 3) with a transepiphyseal type-I fracture had shortening of $2.5 \mathrm{~cm}$ as a result of premature physeal closure but without coxa vara. Another who sustained a type-III basicervical fracture and an ipsilateral fracture of the femoral shaft had $1.7 \mathrm{~cm}$ of overgrowth on the injured side. The third patient, as described above, with a type-II fracture of the femoral neck had shortening of $3 \mathrm{~cm}$ as well as nonunion. 


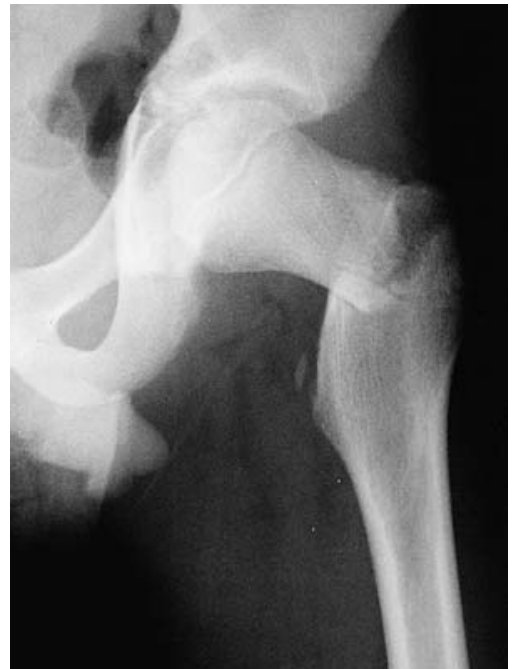

Fig. 3a

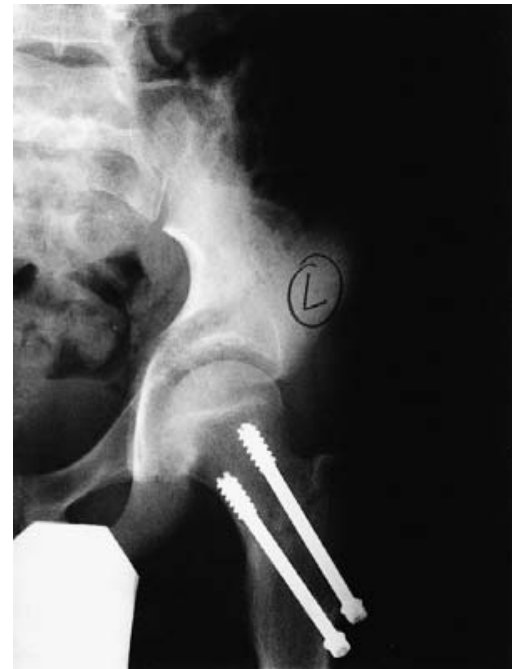

Fig. 3b

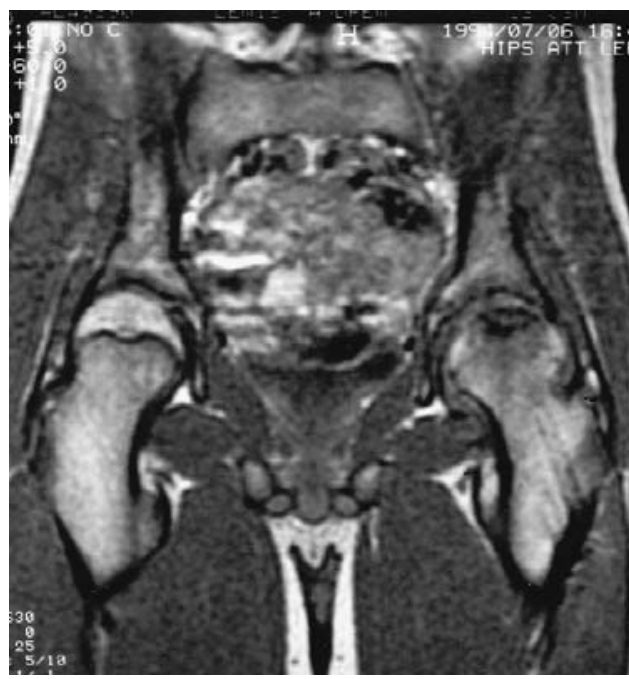

Fig. 3c

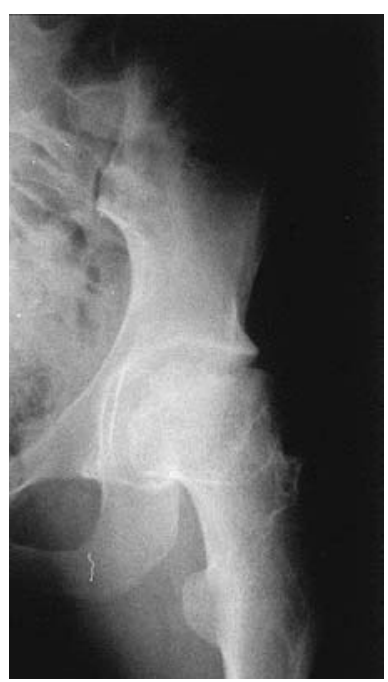

Fig. 3d

Case 6. Figure $3 \mathrm{a}$ - Preoperative anteroposterior radiograph of an 11-year-old boy who sustained a hip fracture after a fall showing a Delbet type-III fracture. Figure $3 b$ - Anteroposterior radiograph showing fixation by two cannulated screws. Figure $3 c-$ MR scan 14 months after fixation showing Ratliff type-II avascular necrosis. Figure $3 \mathrm{~d}$ - Radiograph four years after injury showing incongruity of the femoral head.

\section{Discussion}

Fractures of the hip are uncommon in children, but require careful attention because of the incidence of complications such as avascular necrosis, coxa vara, nonunion, and premature physeal closure. ${ }^{1,2,12}$ We have analysed the longterm results in 18 children who were treated for a displaced fracture of the femoral neck by urgent reduction and internal fixation. Since children are often active and unreliable after operation, and the risks from immobilisation are minimal we used a spica cast in 16 patients for six weeks even although the internal fixation was satisfactory.

Avascular necrosis is the most devastating complication following internal fixation of fractures of the hip in children because of the tenuous blood supply to the femoral head. ${ }^{13}$ There is no effective treatment once avascular necrosis is established. $^{12}$ The incidence ranges from $0 \%{ }^{9}$ to $92 \%,{ }^{12}$ with the most commonly reported being approximately $45 \%,{ }^{1,2,5}$ and varies according to the location of the fracture. Canale and Bourland ${ }^{2}$ reported that the incidence is especially high in Delbet type-I (100\%) and type-II (50\%) and lower in type-III (27\%) and type-IV (14\%) fracture. The degree of displacement of the fracture is thought to be a reliable predictor of avascular necrosis. Canale and Bourland $^{2}$ found that $96 \%$ of fractures in their series which developed avascular necrosis were displaced. Ratliff ${ }^{1}$ 
reported that $71 \%$ of displaced fractures developed complications.

The incidence of avascular necrosis in our study was $6 \%$ (one Delbet type-III). Since avascular necrosis of the hip after an injury in children usually presents within one year, ${ }^{1}$ our minimum follow-up of two years should have detected all potential cases. All the fractures in our study had significant displacement, but were reduced and fixed within 24 hours. Despite the displacement the incidence of avascular necrosis was significantly lower than that reported in most series. While we cannot prove that our management protocol accounted for the low incidence, rapid reduction and rigid internal fixation may have restored the blood supply to the femoral head. Other series ${ }^{9,10}$ based on the same type of protocol have also reported low rates of avascular necrosis. Swiontkowski and Winquist ${ }^{10}$ reported an incidence of $10 \%$ in children with displaced fractures of the hip, treated by urgent open reduction, internal fixation, and anterior capsulotomy. More recently, Cheng and Tang ${ }^{9}$ described 14 patients who had been treated by early internal fixation and aspiration of the hip, none of whom developed avascular necrosis. Only eight patients in their series, however, had displaced fractures (one type-I, five type-II, one type-III and one type-IV).

The requirement for surgical decompression of the capsule remains controversial. Cheng and Tang ${ }^{9}$ and Swiontkowski and Winquist ${ }^{10}$ achieved excellent results with early evacuation of the haematoma. Our incidence of avascular necrosis of $6 \%$ is similar to theirs, although only five of our 18 patients had a capsulotomy. Avascular necrosis occurred in one of our 13 fractures treated without evacuation of the haematoma. Our results suggest that the incidence of avascular necrosis may be low even if the capsule is not surgically opened, as long as the fracture is promptly reduced and stabilised. In some high-energy fractures the hip capsule may be torn at the time of injury, allowing decompression of the capsular haematoma.

In addition to the low rate of avascular necrosis, the incidence of other associated complications was low. Coxa vara did not occur, nonunion was seen in only one patient (6\%) and one fracture (type I) resulted in premature physeal closure. In the three largest series of hip fractures in children in the literature, the rate of coxa vara has been reported as $14 \%,{ }^{1} 21 \%^{2}$ and $32 \%,{ }^{3}$ that of nonunion as $6.5 \%^{2} 27 \%^{3}$ and $33 \%^{1}$ and of premature physeal closure as $20 \%$ and $62 \%$. $^{2}$ The method of treatment seems to be important in the incidence of these complications. Most children with coxa vara, delayed union, or nonunion in the series of Ratliff ${ }^{1}$ and $\mathrm{Lam}^{3}$ were treated without internal fixation. Canale and Bourland ${ }^{2}$ also noted that children treated by a spica cast alone had a greater incidence of coxa vara. They attributed their lower rate of coxa vara and nonunion to the use of internal fixation for all transcervical fractures. Our method of using internal fixation for all displaced fractures of the hip resulted in lower rates of coxa vara, nonunion and premature physeal closure than in previous studies. Our worst result was in an 11-year-old boy (case 6) with a type-III fracture, nonunion and legshortening who was not immobilised in a spica cast because of multiple injuries. Based on this experience, we would now immobilise such a hip for six weeks after surgery.

Of the 18 fractures treated in the last 20 years by our regime, 15 healed without complications. This represents a substantial improvement compared with other large published series of patients with similar fractures. We advise internal fixation of all displaced fractures of the femoral neck even if they can be reduced anatomically. As a precaution, to prevent the child from weight-bearing, we routinely use a spica cast for six weeks after internal fixation. Other surgeons may consider varying the length of the period of immobilisation in a cast depending on the age of the child and the stability of the fracture.

No benefits in any form have been received or will be received from a commercial party related directly or indirectly to the subject of this article.

\section{References}

1. Ratliff AHC. Fractures of the neck of the femur in children. $J$ Bone Joint Surg [Br] 1962;44-B:528.

2. Canale ST, Bourland WL. Fracture of the neck and intertrochanteric region of the femur in children. $J$ Bone Joint Surg [Am] 1977;59-A:431-43

3. Lam SF. Fractures of the neck of the femur in children. $J$ Bone Joint Surg [Am] 1971;53-A:1165-79.

4. Leung PC, Lam SF. Long-term follow-up of children with femoral neck fractures. J Bone Joint Surg [Br] 1986;68-B:537-40.

5. Davison BL, Weinstein SL. Hip fractures in children: a long-term follow-up study. J Pediatr Orthop 1992;12:355-8.

6. Ovesen O, Arreskov J, Bellstrom T. Hip fractures in children: a longterm follow-up of 17 cases. Orthopaedics 1989;12:361-7.

7. Colonna PC. Fractures of the neck of the femur in children. Am J Surg 1929;6:793-7.

8. Boitzy A. Fractures of the proximal femur. In: Weber BG, Brunner C, Freular F, eds. Treatment of fractures in children and adolescents. Berlin: Springer-Verlag, 1980:254-67.

9. Cheng JCY, Tang N. Decompression and stable internal fixation of femoral neck fractures in children can affect the outcome. $J$ Pediatr Orthop 1999;19:338-43.

10. Swiontkowski MF, Winquist RA. Displaced hip fractures in children and adolescents. J Trauma 1986;26:384-8.

11. Larson CB. Rating scale for hip disabilities. Clin Orthop 1964; 31:85-92.

12. Forlin E, Guille Jt, Kumar SJ, Rhee KJ. Complications associated with fracture of the neck of the femur in children. J Pediatr Orthop 1992;12:503-9.

13. Morrissy R. Hip fractures in children. Clin Orthop 1980; 152:202-10. 